\title{
Network pharmacology study on the mechanism of the herb pair of prepared Rehmannia root-Chinese arborvitae kernel for anxiety disorders
}

\author{
Pu-Lin Liu ${ }^{1 \#}$, An-Ran Song ${ }^{1 \#}$, Cheng-Da Dong ${ }^{1}$, Qian Chu ${ }^{1}$, Bao-Lin Xu ${ }^{1}$, Jian-Min Liu ${ }^{2}$, Zhao-Jun Yan ${ }^{2}$ \\ ${ }^{1}$ College of Traditional Chinese Medicine, Shandong University of Traditional Chinese Medicine, Jinan, China; ${ }^{2}$ Department of Psychosomatic \\ Medicine, Affiliated Hospital of Shandong University of Traditional Chinese Medicine, Jinan, China \\ Contributions: (I) Conception and design: ZJ Yan; (II) Administrative support: PL Liu; (III) Provision of study materials or patients: AR Song; (IV) \\ Collection and assembly of data: CD Dong, Q Chu, BL Xu; (V) Data analysis and interpretation: PL Liu, JM Liu; (VI) Manuscript writing: All \\ authors; (VII) Final approval of manuscript: All authors. \\ \#These authors contributed equally to this work. \\ Correspondence to: Zhao-Jun Yan. Affiliated Hospital of Shandong University of Traditional Chinese Medicine, Jingshi Road No.16369, Jinan 250014, \\ China. Email: 361257818@163.com.
}

Background: Although anxiety disorders are one of the most common mental illness in population,
antianxiety drugs often only have single action targets, require long-term use, and are associated with
many adverse reactions and dependencies. Professor Yan Zhaojun from Shandong Provincial Hospital of
Traditional Chinese Medicine (TCM) has applied the modified Renshu Powder, a TCM formula, to treat
anxiety disorders, with satisfactory outcomes. Here, we investigated the mechanism of action of two core
herbs (prepared Rebmannia root and Chinese arborvitae kernel) in the Renshu Powder in the treatment of
anxiety disorders by using network pharmacology approaches. Methods: Candidate compounds of the herb pair of prepared Rebmannia root-Chinese arborvitae kernel were extracted via the Traditional Chinese Medicine Systems Pharmacology (TCMSP) platform. The targets of action of the main compounds were collected using the Swiss TargetPrediction database. Targets associated with anxiety disorders were retrieved from DisGeNET, Online Mendelian Inheritance in Man (OMIM), DrugBank, GeneCards, and Comparative Toxicogenomics Database (CTD) databases. The compound-target interaction network was constructed by Cytoscape 3.7.2 software, and the protein-protein interaction (PPI) network was constructed using the Search Tool for the Retrieval of Interacting Genes/Proteins (STRING) platform. Gene Ontology (GO) and Kyoto Encyclopedia of Genes and Genomes (KEGG) analyses the data by using Metascape.

Results: The main active compounds of the herb pair included arachidonic acid, stigmasterol, and betasitosterol. The key targets included Nitric Oxide Synthase 3 (NOS3), Epidermal growth factor (EGF), Prostaglandin-Endoperoxide Synthase 2 (PTGS2), Caspase 3 (CASP3), Mitogen-Activated Protein Kinase 1 (MAPK1), Peroxisome proliferator-activated receptor gamma (PPARG), RELA Proto-Oncogene, NFKB Subunit (RELA), Estrogen Receptor 1 (ESR1), Solute Carrier Family 6 Member 4 (SLC6A4), and Phosphatase and Tensin homolog deleted on chromosome 10 (PTEN). Anxiety disorder-related GO analysis mainly involved synaptic signaling, neurotransmitter receptor activity, and $G$ protein-coupled neurotransmitter receptor activity. The KEGG pathways involved neuroactive ligand-receptor interaction, serotonergic synapse, PI3K/AKT/mTOR signaling pathway, and MAPK signaling pathway.

Conclusions: The mechanism of action of the prepared Rebmannia root-Chinese arborvitae kernel in treating anxiety disorders involves multiple ingredients, multiple targets, and pathways.

Keywords: Prepared Rebmannia root; Chinese arborvitae kernel; anxiety disorders; network pharmacology; mechanism of action 
Submitted Jan 27, 2021. Accepted for publication Mar 23, 2021.

doi: 10.21037/apm-21-531

View this article at: http://dx.doi.org/10.21037/apm-21-531

\section{Introduction}

Anxiety reflects the anticipation of future threats and is a normal emotional experience; however, when this emotion is severe enough to affect behavior or cause distress, it can develop into an anxiety disorder (1). Anxiety disorders are one of the most common mental problems in the adults, with a prevalence rate of $7.3 \%$ worldwide (2) and $7.6 \%$ in China (3). Thus, it has become a social problem that cannot be ignored. According to the American Psychiatric Association's Diagnostic and Statistical Manual of Mental Disorders, Fifth Edition (DSM-5), anxiety disorders include separation anxiety disorder, selective mutism, specific phobia, social anxiety disorder, panic disorder, agoraphobia, generalized anxiety disorder, substance-induced anxiety, anxiety disorder due to other medical conditions, and unspecified/undefined anxiety disorder (4). The COVID-19 pandemic, which was first identified in December 2019, has been found to be associated with high incidences of psychological symptoms, such as anxiety and depression, which has raised public concern about mental health (5). Another study has shown that patients can be plagued by sleep disturbances, anxiety, or depression even 6 months after being discharged from the hospital with a cure (6).

Antianxiety drugs often only have single-action targets, require long-term use, and are associated with many adverse reactions and dependencies (7). In contrast, guided by the traditional Chinese medicine (TCM) holistic concept, the TCM prescriptions can be used to treat diseases with their multiple compounds, multiple targets of action, and multiple pathways, and share the advantages of fewer adverse reactions and higher patient compliance (8). Similarly ,TCM has certain characteristics. Many TCM drugs have shown efficacies in reducing the negative effects of anxiety symptoms and in enhancing mental health (9). The ancient Chinese formula Renshu Powder (initially seen in the Introduction to Medicine, Li Ting, Ming dynasty) has long been used to treat mental and physical symptoms caused by anxiety and fear. For decades, Professor Yan Zhaojun from Shandong Provincial Hospital of Traditional Chinese Medicine has applied the modified Renshu Powder to treat anxiety disorders, with satisfactory outcomes (10). The combination of prepared Rebmannia root and Chinese arborvitae kernel is the core drug pair in the formula.

Based on the theories of system biology, network pharmacology affords a powerful and practical research method (11). By utilizing multidisciplinary theories, it predicts the mechanism of action of a specific compound in treating a specific disease by screening the potential bioactive components, targets, and pathways. The principles of network pharmacology are consistent with TCM theories. Recent studies have shown that the possible mechanisms of COVID-19 treatment by the five recommended prescriptions are regulation of immune system, anti-inflammatory, antiviral, regulation of cell apoptosis, anti-pulmonary fibrosis, myocardial protection, etc. At the same time, $\beta$-sitosterol, quercetin and kaempferol may be involved in the treatment of COVID-19 at various stages, showing great potential anti-epidemic activity (12). Here we predicted the potential pharmacological mechanisms of the action of the herb pair of prepared Rebmannia root and Chinese arborvitae kernel, especially the targets and their compounds, in the treatment of anxiety disorders by using network pharmacology approaches, with a view to promote the global use of TCMbased antianxiety medications. We present the following article in accordance with the MDAR checklist (available at http://dx.doi.org/10.21037/apm-21-531).

\section{Methods}

\section{Main active compounds and targets of the TCM drugs}

The chemical composition of prepared Rebmannia root and Chinese arborvitae kernel was searched in the TCMSP database (http://tcmspw.com/tcmsp.php) (13), and the main active compounds and target proteins were filtered based on parameters including oral bioavailability $(\mathrm{OB}) \geq 30 \%$, druglike properties (DL) $\geq 0.18$, and blood-brain barrier (BBB) $\geq-0.30$ (14). The SMILES codes of all active compounds were confirmed through PubChem (http://pubchem.ncbi. nih.gov) (15). The targets of action of the main active components were obtained using the SwissTargetPrediction database (http://www.swisstargetprediction.ch/) (16). The target proteins were genetically normalized using the Uniprot database (http://www.uniprot.org/) and deduplicated to obtain the gene names of the targets of 
the main active compound of the drug pair of prepared Rebmannia root-Chinese arborvitae kernel. The main active compounds of the herb pair and their targets were imported into the Cytoscape software 3.7.2 (17) to construct a visual network of TCM Drugs-ingredients-targets. The study was conducted in accordance with the Declaration of Helsinki (as revised in 2013).

\section{Collection of disease-related targets}

With Anxiety Disorders as the keyword, searching for human genes in databases including DisGeNET (https:// www.disgenet.org/) (18), Online Mendelian Inheritance in Man (OMIM) (http://www.omim.org) (19), Drugbank (https://www.drugbank.ca) (20), GeneCards (https://www. genecards.org/) (21), and Comparative Toxicogenomics Database (CTD) (http://ctdbase.org/) (22). After literature review and data deduplication/integration, the anxiety disorder-related targets were obtained. The targets of the main active compounds in the herb pair were mapped to the disease-related targets, and the intersecting genes (i.e., the predicted targets of the herb pair at which point anxiety disorder are intervention occurs) were obtained.

\section{Protein-protein network construction and analysis}

The predicted targets were imported into the Search Tool for the Retrieval of Interacting Genes/Proteins (STRING) platform (https://string-db.org/cgi/input.pl) (23) for protein-protein interaction (PPI) network construction, and the minimum required interaction score was set to 0.400. The PPI network was constructed by importing the STRING platform data files into Cytoscape software 3.7.2, and topology analysis was performed by using the Network Analyzer tool. The topology network was analyzed based on 3 parameters: Degree, Betweenness Centrality (BC), and Closeness Centrality (CC)". After 3 sessions of filtering (criteria: parameter values higher than the medians), the core targets were obtained.

\section{Molecular docking validation}

Receptor-ligand docking simulations were performed using Autodock Vina 1.1.2 program (Trott and Olson, 2010) for the top 3 key compounds of Degree and the top 3 core targets obtained from the filtering, with the docking results between the first-line therapeutic drug sertraline hydrochloride (24) and the core targets being used as a control.

\section{Targets and patbways enrichment analyses}

The predicted anxiety-intervening targets were filtered using the Metascape database (25). For targets that met the filtering criteria of $\mathrm{P}<0.01$, Gene Ontology (GO)-Biological Process and Kyoto Encyclopedia of Genes and Genomes (KEGG) pathway enrichment analyses were performed, and the results were visualized in Cytoscape.

\section{Establishing the TCM drugs-ingredients-diseases-targets- patbways network}

The main active ingredients, diseases, predicted targets, and major pathways of the herb pair were imported into the TCM drugs-ingredients-diseases-targets-pathways network, in order to more visually demonstrate the multitarget and multipathway characteristics of the prepared pair of Rebmannia root-Chinese arborvitae kernel for intervention of anxiety disorders.

\section{Statistical analysis}

The selected targets obtained in this study were analyzed by rigorous statistics. We analysed the topological data by Cytoscape version 3.6.0. Database for Annotation, Visualization, and Integrated Discovery (DAVID) version 6.8. The STRING platform data was imported into Cytoscape software 3.7.2 to construct the PPI network. The topology analysis was performed by using the Network Analyzer tool. All the relevant data after statistical analysis are shown in "Results" Section below.

\section{Results}

\section{Main active compounds and targets of the TCM drugs}

Six main active compounds of prepared Rebmannia root and Chinese arborvitae kernel were obtained by filtering via TCMSP. Among them, there were 2 active compounds in prepared Rebmannia root and 5 in Chinese arborvitae kernel. Beta-sitosterol was a shared active compound in these 2 herbs (Table 1). After gene normalization and removal of duplicate values, 102 action targets were obtained. The main active compounds of the herb pair and their targets were imported into the Cytoscape software 
Table 1 The main active compounds of the herb pair of prepared Rehmannia root and Chinese arborvitae kernel

\begin{tabular}{|c|c|c|c|c|c|c|}
\hline Source & Mol ID & Molecule name & OB (\%) & BBB & $\mathrm{DL}$ & Degree value \\
\hline Prepared Rehmannia root & MOL000449 & Stigmasterol & 43.83 & 1.00 & 0.76 & 45 \\
\hline Chinese arborvitae kernel & MOL000359 & Beta-sitosterol & 36.91 & 0.87 & 0.75 & 19 \\
\hline Chinese arborvitae kernel & MOL001439 & Arachidonic acid & 45.57 & 0.58 & 0.20 & 50 \\
\hline Chinese arborvitae kernel & MOL003927 & 11,14,17-eicosatrienoic acid & 44.11 & 0.88 & 0.20 & 18 \\
\hline Chinese arborvitae kernel & MOL008153 & 5Z-eicosenoic acid & 30.70 & 0.89 & 0.20 & 18 \\
\hline
\end{tabular}

$\mathrm{OB}$, oral bioavailability; BBB, blood-brain barrier; DL, drug-like.

to construct a visual network of TCM drugs-ingredientstargets (Figure 1). The network topology analysis using the Network Analyzer plug-in showed that there were 110 nodes and 167 edges, with an average degrees of freedom of 3.036. Among these active compounds, arachidonic acid, stigmasterol, and beta-sitosterol had the highest Degree values, suggesting they were the key active compounds in the herb pair.

\section{Collection of disease targets}

Disease-related targets were searched in DisGeNET, OMIM, Drugbank, GeneCards, and CTD databases, and a total of 4,790 targets were obtained after removing duplicate values. The drug pair targets were mapped to the disease targets, and 70 predicted targets were obtained.

\section{PPI network construction and analysis}

These 70 predicted targets were loaded into the STRING platform to obtain the predicted PPI relationship network, which had 70 nodes and 358 edges, with a mean Degree value of 10.2. The data were imported into Cytoscape for topological analysis, and the PPI network yielded from this is presented in Figure 2. The core targets were identified on the basis of the Degree value, BC, and CC and arranged according to the Degree value (Figure 3). The top 10 targets were NOS3, EGF, PTGS2, CASP3, MAPK1, PPARG, RELA, ESR1, SLC6A4, and PTEN, indicating these targets play important roles in the treatment of anxiety disorders.

\section{Molecular docking validation}

The top 3 active compounds ranked by Degree value were selected to dock with the top 3 core targets, and sertraline hydrochloride was used as a positive control. The results show that the binding energies of all molecules to proteins are less than 0 , which indicates that the ligand can spontaneously bind to the receptor; the binding energies of stigmasterol and beta-sitosterol to the core target were less than $-5.0 \mathrm{~kJ} / \mathrm{mol}$, and their binding energies for docking with sertraline hydrochloride were quite similar. Thus, the proper core targets and ligands were selected. Stigmasterol and beta-sitosterol had the optimal binding energies to NOS3, as shown in Table 2 and Figures 4 and 5, in which the red dashed lines represent hydrogen bonds, indicating that stigmasterol forms hydrogen bonding with NOS3 at the active site ARG-365 and beta-sitosterol forms hydrogen bonding with NOS3 at the active site TRP-356.

\section{Targets and pathways enrichment analyses}

With a filtering condition of $\mathrm{P}<0.01$, the predicted targets underwent enrichment analyses in Metascape software. A total of 53 GO terms were obtained, consisting of 20 biological process (BP) terms, 14 cell composition (CC) terms, and 19 molecular functions (MF) terms, as shown in Figure 6. Enrichment analysis of KEGG pathway showed 80 KEGG pathways, and the top 20 pathways are visualized with a bar graph (Figure 7).

\section{Establishing TCM drugs-ingredients-diseases-targets- pathways network}

The herb pair and its main active compounds, diseases, predicted targets, and main pathways were imported into Cytoscape software to draw the TCM drugs-ingredientsdiseases-targets-pathways network, with an attempt 


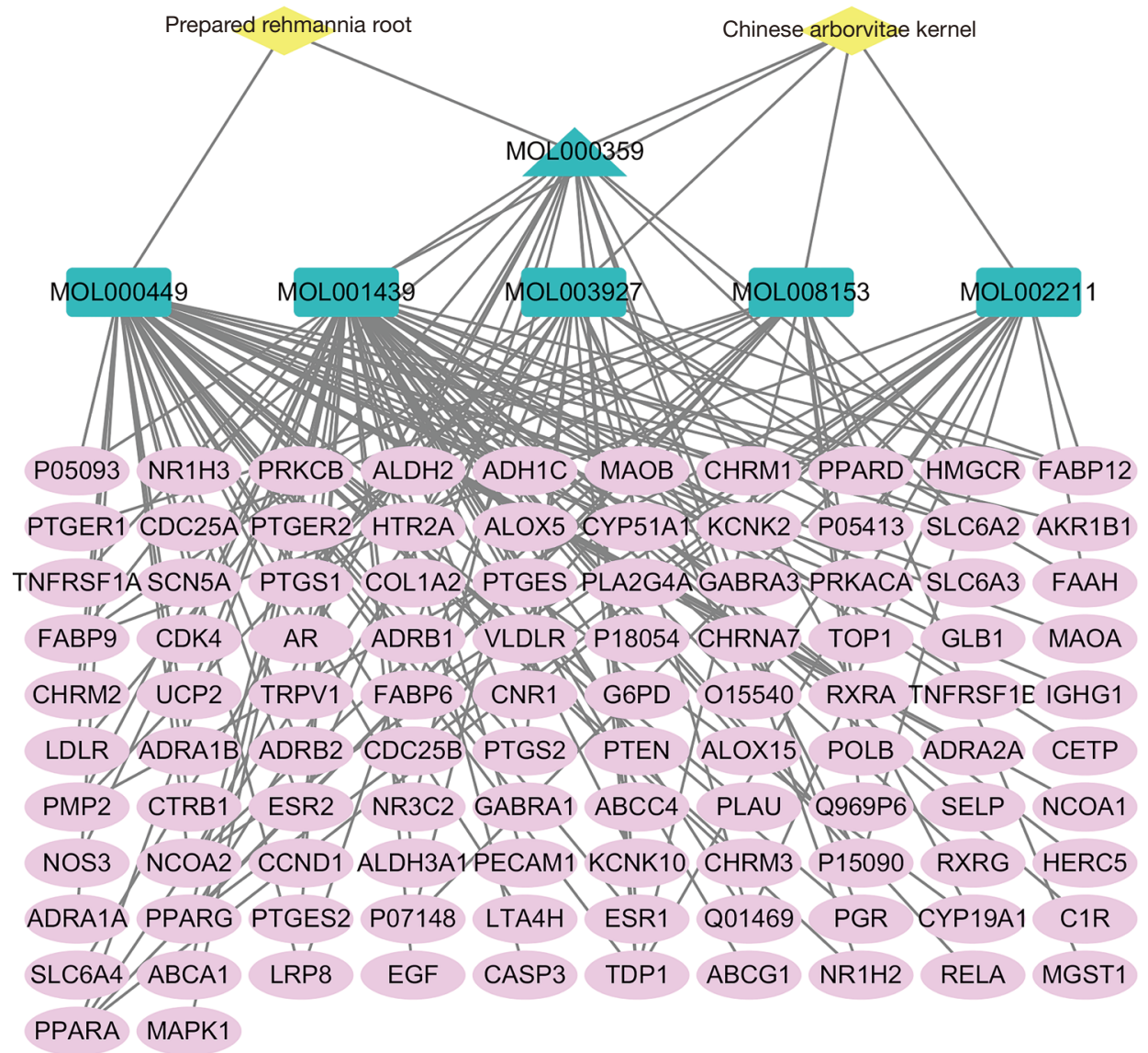

Figure 1 TCM drugs-ingredients-targets network. TCM, Traditional Chinese Medicine.

to demonstrate the multicompound, multitarget, and multipathway characteristics of the herb pair. The results are shown in Figure 8.

\section{Discussion}

Experimental and clinical studies have demonstrated that both genetic and environmental factors contribute to the risk of developing an anxiety disorder (26). The common triggers for anxiety disorders include neurotransmitters, neuroendocrine function, neuroanatomy, immune disorders, stressful psychological events, and drug addiction and withdrawal (27). These influencing factors are also involved in the targets and pathways by which prepared Rebmannia root and Chinese arborvitae kernel treat anxiety disorders.

It has been confirmed that prepared Rebmannia root can exert its anxiolytic effect through enhancing GABAAR1 expression, increasing central $\gamma$-aminobutyric acid (GABA) level, and reducing $\mathrm{N}$-methyl-D-aspartate receptor 1 expression by decreasing glutamate level (28). Filtering in our current study revealed that arachidonic acid, stigmasterol, and beta-sitosterol were the key active compounds in the herb pair prepared Rehmannia root and Chinese arborvitae kernel. Arachidonic acid (AA) is one of the most abundant and widely distributed polyunsaturated fatty acids (PUFA) in mammals and also one of the essential fatty acids in humans. It is catalyzed by phospholipase A2 (PLA2) and can generate various metabolites through cyclooxygenase (COX), lipoxygenase (LOX), and cytochrome $\mathrm{P} 450$ (CYP450) pathways, playing a wide range of important physiological roles; in particular, it has unique biological activities in lowering blood lipids; inhibiting platelet aggregation; fighting against inflammation, cancer, and lipid oxidation; and promoting the development of brain tissue (29). Research has shown that stigmasterol exerts its neuroprotective effects by downregulating 


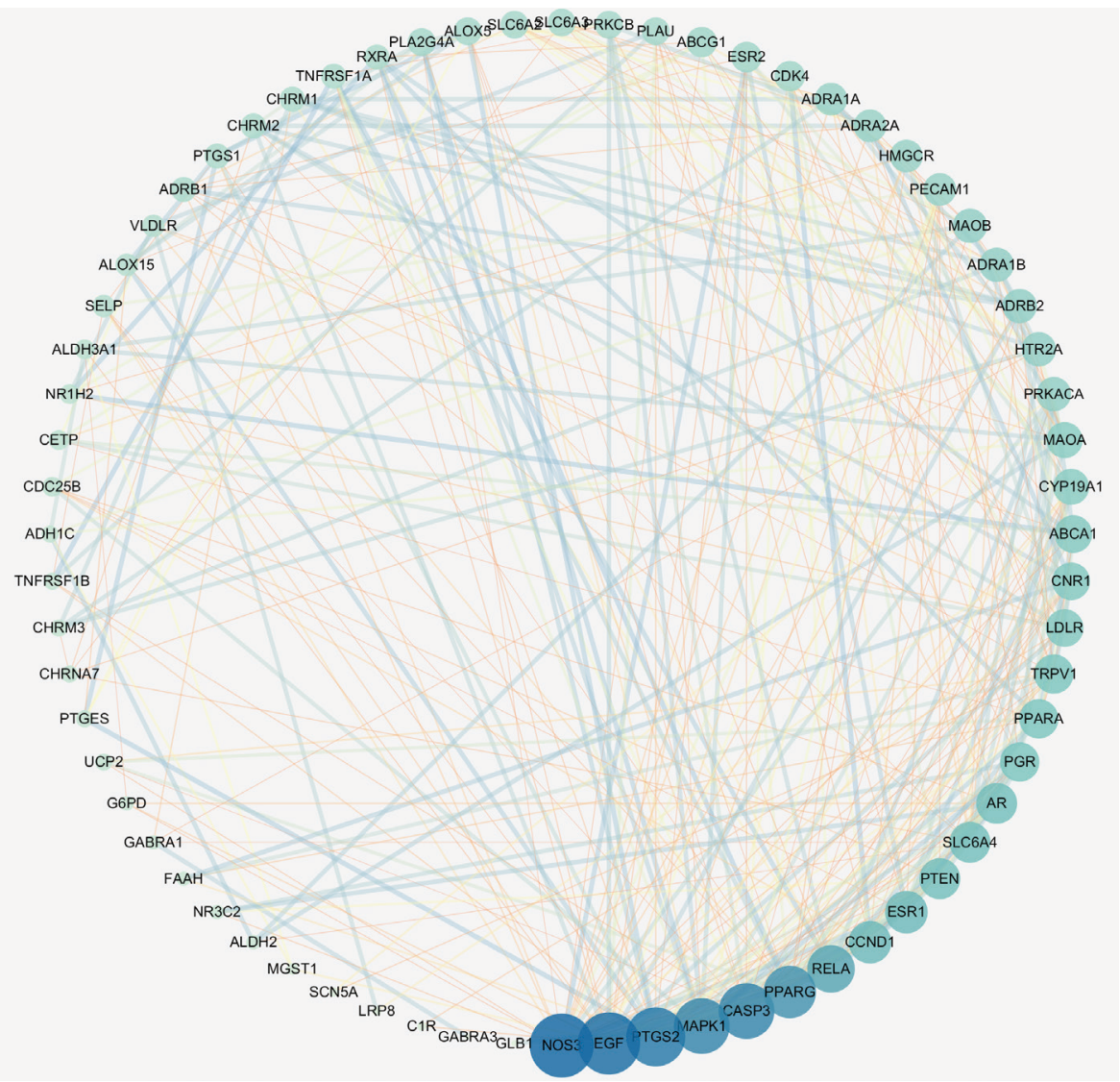

Figure 2 PPI network. PPI, protein-protein interaction.

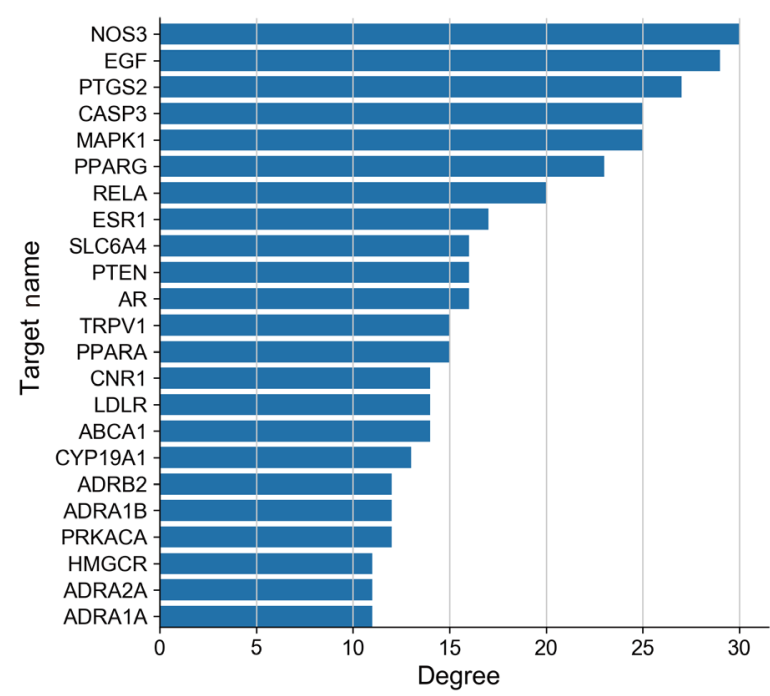

Figure 3 Core targets.
GluN2B-mediated excitotoxicity and reducing oxidative stress, showing therapeutic promise for cerebrovascular and neurodegenerative diseases (30). Beta-sitosterol has sedative and anxiolytic effects (31), and its mechanism of action may be related to the GABA/benzodiazepine receptor complex (32). Monoamine neurotransmitters, such as 5-HT, norepinephrine (NE), and dopamine (DA), have been suggested to play important roles in the pathogenesis of neurological and psychiatric disorders, and $\beta$-sitosterol may also exert its anxiolytic effect by mediating DA, 5-HT, and NE (33).

In our current study, topological analysis revealed that the key targets of the herb pair of prepared Rebmannia root and Chinese arborvitae kernel for anxiety disorders were NOS3, EGF, PTGS2, CASP3, MAPK1, PPARG, RELA, ESR1, SLC6A4, and PTEN. The nitric oxide synthase 3 (NOS3) gene is located in chromosome region $7 \mathrm{p} 35-\mathrm{p} 36$. Physiologically, nitric oxide (NO) in blood vessels is mainly synthesized by NOS3. NOS is mainly expressed in neurons 
Table 2 Docking of the main active compounds with the core target protein molecules

\begin{tabular}{lccc}
\hline Active compound & Target protein & PDB (Protein Data Bank) ID & Binding energy $\left(\mathrm{kJ}^{\mathrm{m}} \mathrm{mol}^{-1}\right)$ \\
\hline Arachidonic acid & NOS3 & $4 \mathrm{D} 1 \mathrm{P}$ & -6.3 \\
& EGF & $1 \mathrm{NQL}$ & -4.2 \\
Stigmasterol & PTGS2 & $5 \mathrm{~F} 19$ & -6.7 \\
& NOS3 & $4 \mathrm{D} 1 \mathrm{P}$ & -9.2 \\
& EGF & $1 \mathrm{NQL}$ & -5.9 \\
Beta-sitosterol & PTGS2 & $5 \mathrm{~F} 19$ & -8.7 \\
& NOS3 & $4 \mathrm{D} 1 \mathrm{P}$ & -9.2 \\
& EGF & $1 \mathrm{NQL}$ & -5.5 \\
Sertraline & PTGS2 & $5 \mathrm{~F} 19$ & -7.1 \\
& NOS3 & $4 \mathrm{D} 1 \mathrm{P}$ & -9.0 \\
& EGF & $1 \mathrm{NQL}$ & -5.2 \\
\hline
\end{tabular}

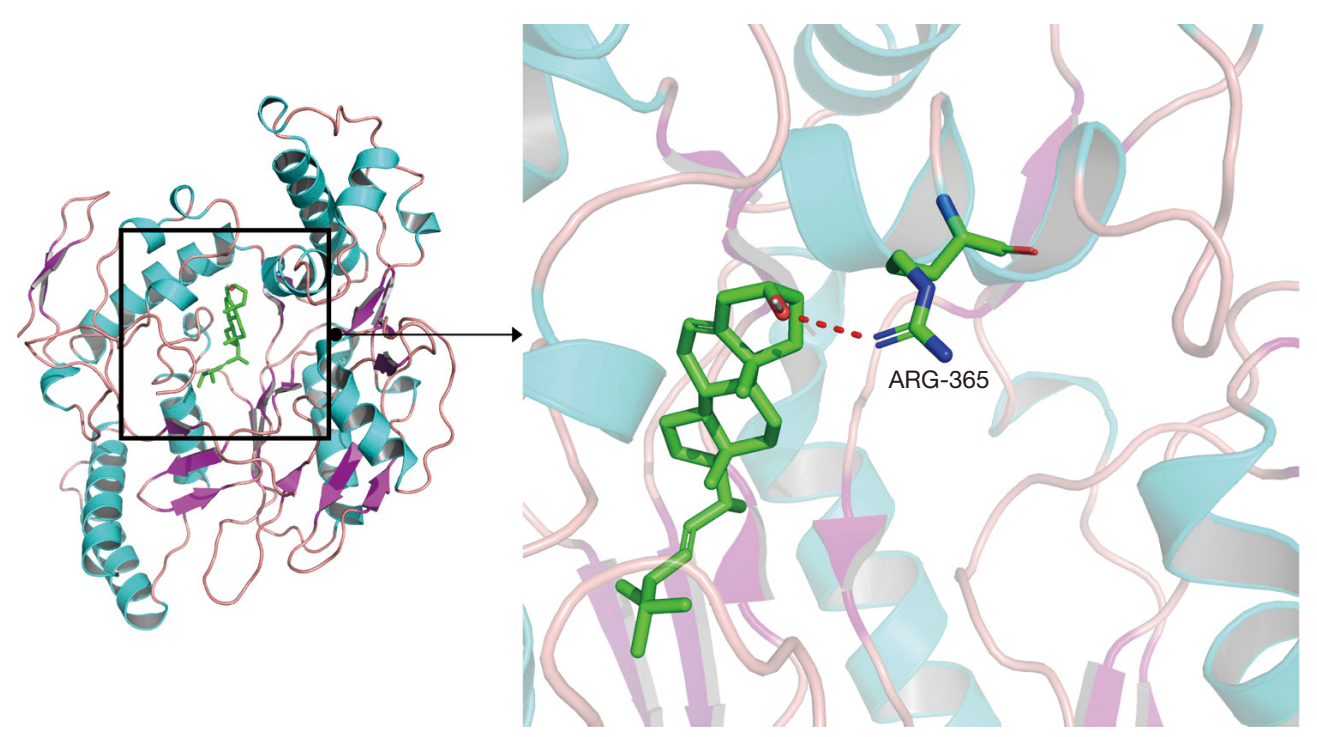

Figure 4 Molecular docking of stigmasterol with NOS3.

and differentially distributed in different regions of the brain; abnormal NOS3 gene expression induces neuronal and glial degeneration in the brain (34). Malformations of the cerebral vasculature are associated with reduced NOS3 expression. Decreased NOS3 expression in cerebral vasculature is associated with the risk of vascular lesions, vascular smooth muscle cell apoptosis, and amyloid plaque formation (35). As a physiologically active low-molecularweight polypeptide, epidermal growth factor $(E G F)$ plays a special role in regulating cell growth, proliferation, and differentiation. Its pathophysiological roles in various diseases have increasingly been recognized. $E G F$ is closely related to cognitive function, as it is a potent mitogen that promotes the growth and development of the nervous system while protecting and nourishing neurons and dopamine when oxidative damage to neurons occurs (36). The PTGS2 gene is a core enzyme in prostaglandin biosynthesis and has been shown to play 


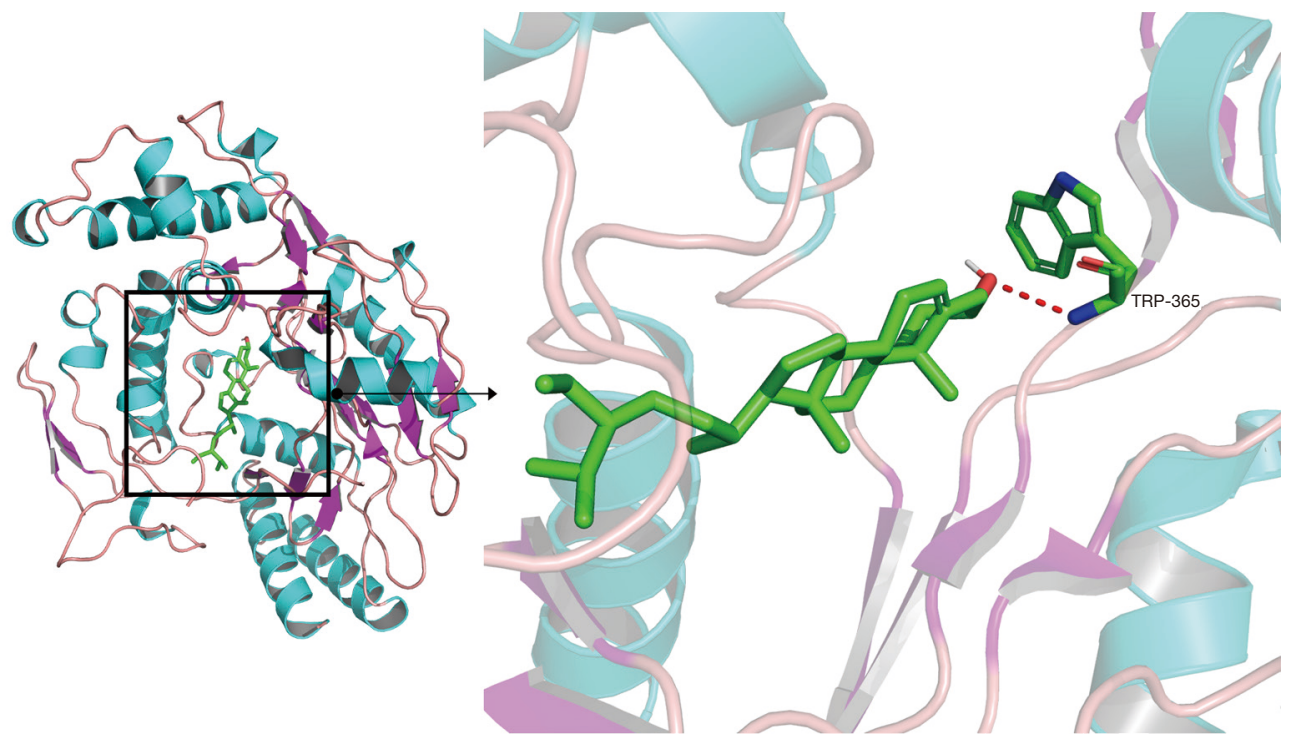

Figure 5 Molecular docking of beta-sitosterol with NOS3.
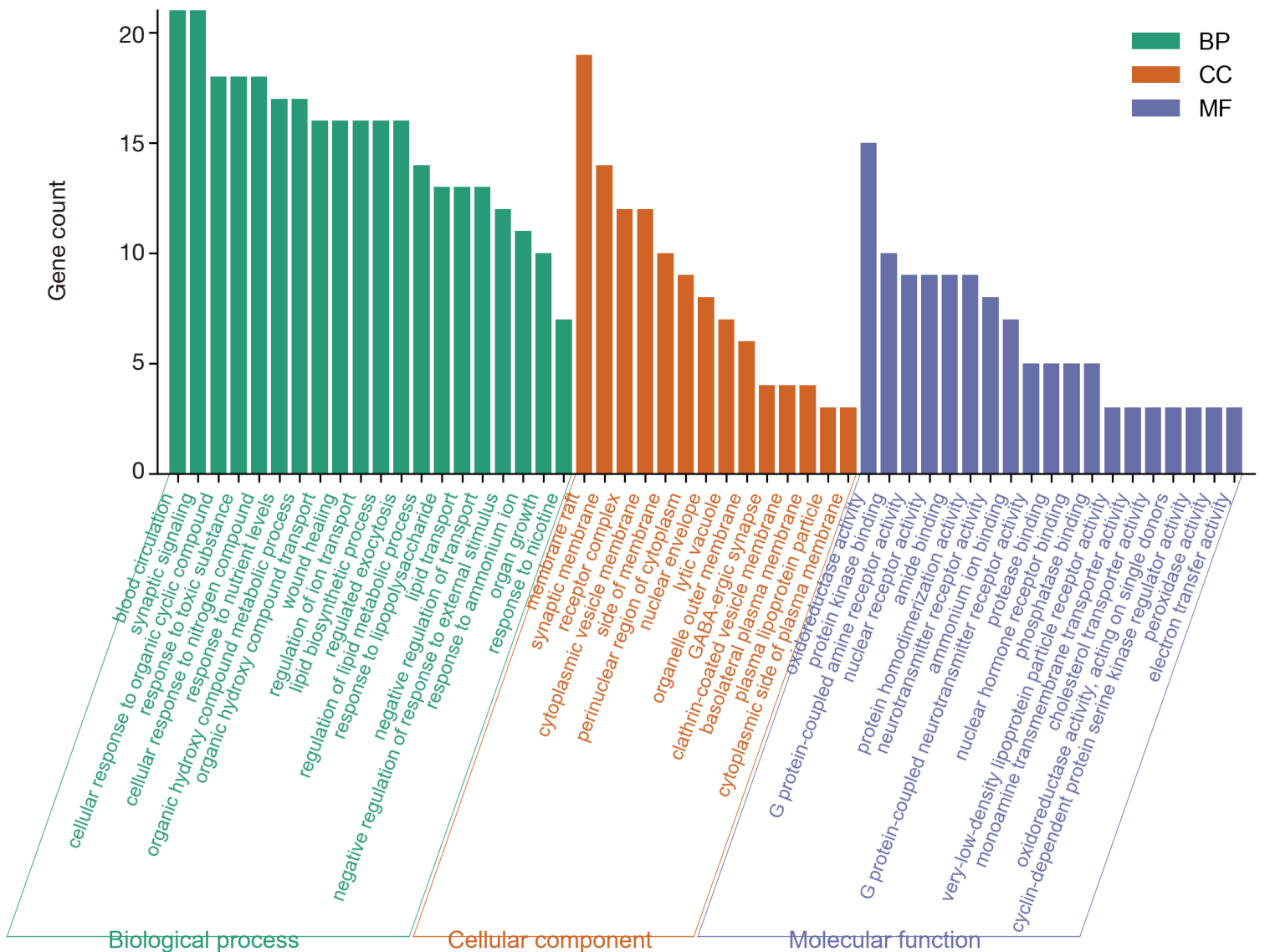

Figure 6 Gene Ontology enrichment analysis-biological process. 


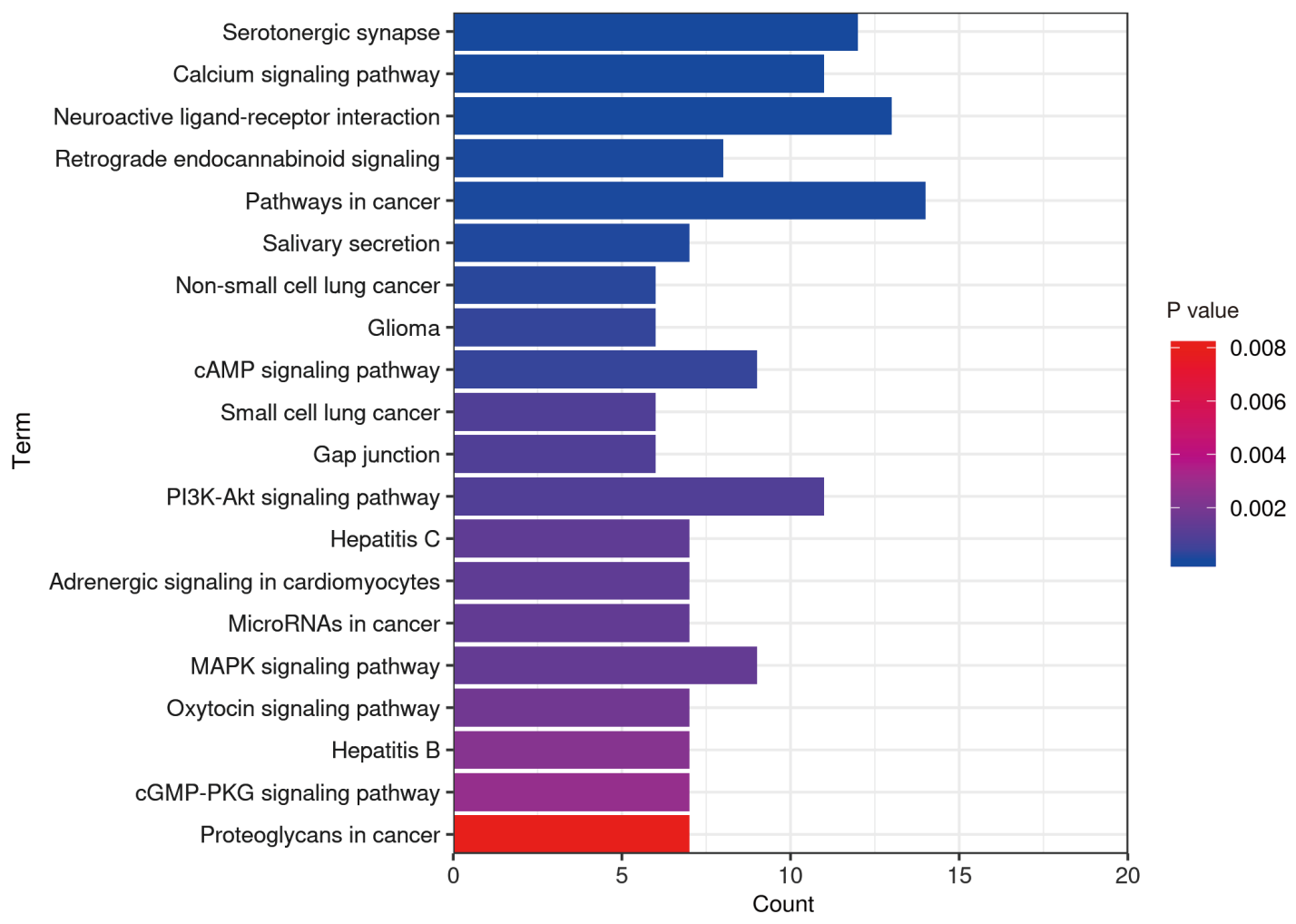

Figure 7 KEGG pathway enrichment analyses. KEGG, Kyoto Encyclopedia of Genes and Genomes.

an important role in the development of Alzheimer's disease (37). PTGS2 may increase the production of inflammatory chemokines and cytokines by activating its downstream pathways (e.g., PI3-K/AKT and PKA/ CREB pathways), thus promoting neuronal apoptosis and inflammation and inhibiting neurite outgrowth (38). Mitogen-activated protein kinase 1 (MAPK1), an effector kinase of the MAP kinase signaling pathway, is controlled by cell proliferation, differentiation, apoptosis, and angiogenesis (39). Anxiety disorder comorbidity, as a risk factor for suicide, was found to be significantly associated with variations in MAPK1 polymorphisms (40). High levels of peroxisome proliferator-activated receptors (PPAR) have been found in the hippocampus and amygdala of the brains of patients with anxiety disorders. PPAR group includes three isoforms encoded by the PPARG, PPARA, and PPARD genes, with the gamma type (i.e., $P P A R G$ ) showing the highest expression in the central nervous system; studies have identified at least 14 PPAR-regulated proteins associated with anxiety disorders, suggesting that PPAR might be involved in neuroinflammatory protection (41). Estrogen is thought to play a key role in anxiety. Estrogen receptor 1 (ESR1) polymorphisms are associated with the risk of anxiety disorders (42), and there is a strong association between ESR1 variants and cognitive outcomes (43). Alterations in serotonin 5 -hydroxytryptamine (5-HT) neurotransmission and peripheral immune activation have been shown to be strongly associated with a variety of psychiatric disorders (44). SLC6A4 is a serotonin transporter gene. Studies have shown that there are many predisposing factors for affective disorder, such as epigenetic processes including SLC6A4 promoter methylation and microRNA silencing. (45). The tumor suppressor PTEN is a vital homeostatic regulator, by virtue of its lipid phosphatase activity against phosphatidylinositol 3,4,5-trisphosphate [PI $(3,4,5) \mathrm{P} 3]$, which downregulates the PI3K/AKT/mTOR prosurvival signaling (46). PTEN plays an important role in the development of neurons. In addition, it also plays a role in synaptic plasticity in adulthood. Studies have shown that the synaptic function of PTEN in amygdala is closely related to specific behavioral characteristics (47).

In our current study, GO enrichment analysis was performed to describe the intracellular properties of core 


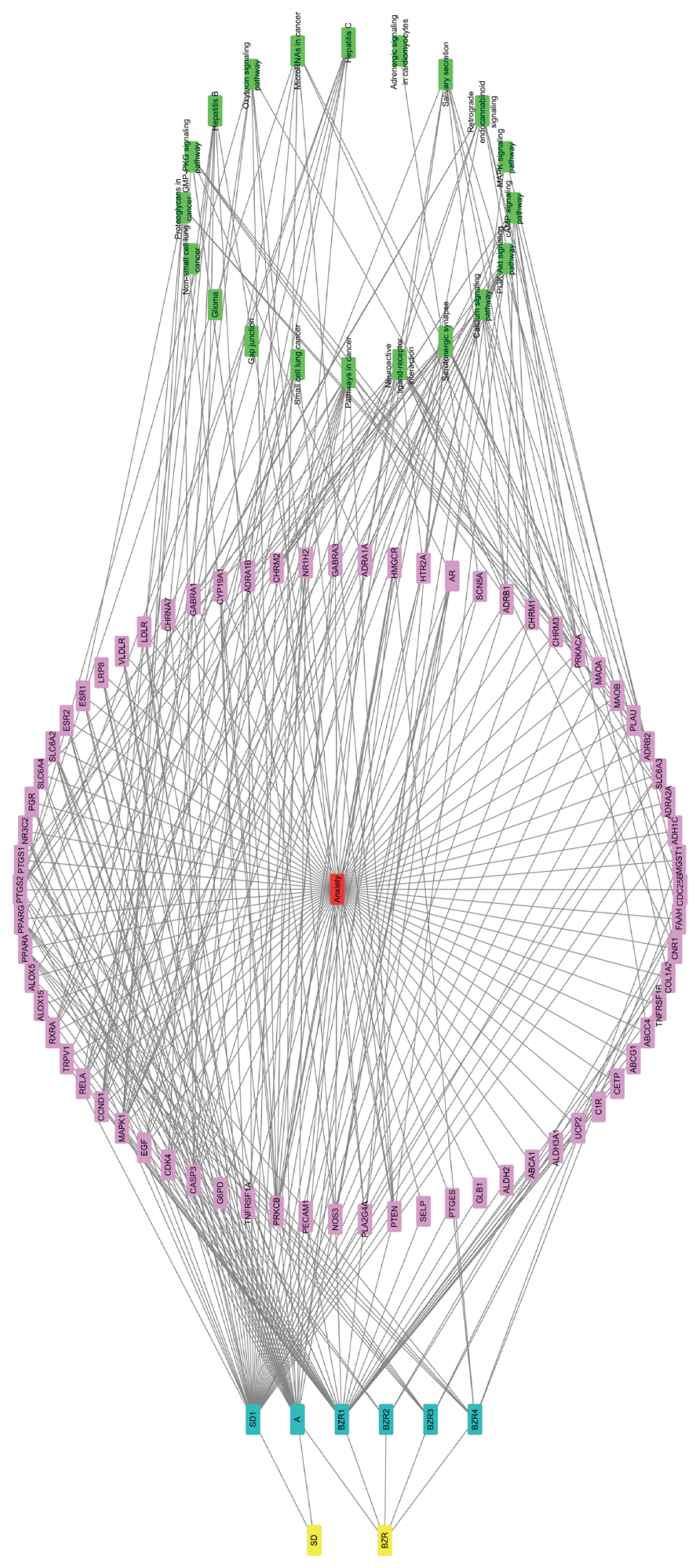

Oت 
target proteins, where biological processes, including blood circulation, synaptic signaling, cellular response to organic cyclic compound, response to toxic substance, cellular response to nitrogen compound, response to nutrient levels, and organic hydroxy compound metabolic process, were found to be involved in the therapeutic effects of prepared Rebmannia root and Chinese arborvitae kernel on anxiety disorders. The related molecular functions, including oxidoreductase activity, protein kinase binding, G protein-coupled amine receptor activity, nuclear receptor activity, amide binding, protein homodimerization activity, neurotransmitter receptor activity, and ammonium ion binding, might enable the herb pair to exert its antianxiety activity. Furthermore, cellular localization showed that the mechanism sites were the membrane raft, synaptic membrane, receptor complex, cyptoplasmic vesicle membrane, side of membrane, perinuclear region of the cytoplasm, and other cellular environments.

KEGG enrichment pathways mainly involved signaling pathways (e.g., PI3K-Akt signaling pathway, mTOR signaling pathway, cAMP signaling pathway, MAPK signaling pathway, TNF signaling pathway, and VEGF signaling pathway), neurological system (e.g., neuroactive ligand-receptor interaction and serotonergic synapse), endocrine system (e.g., thyroid hormone signaling pathway, estrogen signaling pathway, and estrogen signaling pathway), and drug dependence (e.g., cocaine addiction and amphetamine addiction); in addition, the immune system, cellular processes, cancer, and amino acid metabolism were also involved.

Neuroactive ligand-receptor interaction signaling pathways are closely related with learning and memory and play key roles in neuronal processes, such as neural plasticity and synaptic function (48). In psychological stress-induced susceptible mice versus resilient mice, the differentially expressed genes (DEGs) were upregulated in neuroactive ligand-receptor interaction, PI3K-Akt, VEGF, Ras, and chemokine pathways, and downregulated in cGMP-PKG, B cell receptor, and NOD-like receptor pathways (49). The $\mathrm{PI} 3 \mathrm{~K} / \mathrm{AKT} / \mathrm{mTOR}$ signaling pathway is one of the classical pathways regulating the cell cycle. It is widely present in neuronal cells and plays an important role in the regulation of cell division, differentiation, and survival, as well as tumorigenesis. It is closely associated with a variety of mood disorders and neurological diseases. The PI3K/Akt signaling pathway is a downstream signaling pathway of $\mathrm{BDNF} / \operatorname{TrkB}$ and is involved in disease pathogenesis through processes such as neurotrophic factor release, glutamatergic system, hippocampal neuronal apoptosis, mitochondrial function, glucose/lipid metabolism, and cerebral angiogenesis (50-52). The mTOR signaling pathway involves the proliferation of neural stem cells, regulation of neural circuits, and regulation of circadian rhythms. It regulates memory and learning behaviors through synaptic plasticity (53). Recent studies have confirmed that ketamine is a glutamatergic $\mathrm{N}$-methyl-D-aspartate receptor (NMDA-R) antagonist, which has the characteristics of fast onset and lasting effect in the process of antidepressant. It is therefore assumed that depression is closely associated with the mTOR pathway, and the mTOR pathway may play a key role in depression treatment (54). Another experiment confirmed that early-life stress alters synaptic plasticity by inhibiting the hippocampal mTOR pathway, which may result in increased anxiety-like behaviors and impaired cognitive function (55); anxiety disorders are associated with inflammation. Long-term stress environment can destroy the function of hypothalamus pituitary adrenal axis and the autonomic nervous system, which may induce systemic proinflammatory diseases. Systemic inflammation can enter the brain and increase levels of pro-inflammatory cytokines, which have been shown to promote both direct and indirect neurotoxic effects (56). thus, the mTOR pathway is closely related to anxiety disorders and may be a new direction for antianxiety drug research. The MAPK/ERK signaling pathway is a mechanism that mediates the transmission of extracellular signals to their intracellular targets. It affects the emotional function, social behavior, and spatial memory in humans and is a key element of the neuroinflammatory pathway triggered by glial cells during the development of neurodegenerative diseases (57). Acute local blockade of this MAPK signaling pathway in hypothalamic paraventricular nucleus (PVN) may cause a profound anxiogenic phenotype (58). Phosphodiesterase (PDEs) is an enzyme involved in camp and cGMP (two key second messengers) homeostasis. Phosphodiesterase 2 (PDE2) is involved in cGMP PKG signal transduction, which is highly expressed in limbic brain regions (including hippocampus and amygdala), which may become a breakthrough in the treatment of depression and anxiety $(59,60)$.

Alterations in various behaviors (including appetite, mood, sleep, and cognitive function) in patients with anxiety disorders have been associated with the serotonergic system, and a growing body of research supports a role for dysfunction of serotoninergic, noradrenergic, and dopaminergic systems in the neurobiological processes involved in major depression disorder and anxiety 
disorders (61). Estrogen promotes synapse formation, induces growth factor production, protects against oxidative stress, regulates cognition- and mood-related neurotransmitters such as 5-HT, norepinephrine (NE), and acetylcholine, and is associated with learning-, memory-, and anxiety-related behaviors (43). The amygdala is an important structure for anxiety and fear responses; research has confirmed that oxytocin can influence anxiety levels by regulating amygdala activation (62). Thyroid hormones can facilitate intracellular pathways of signaling and affect the neurotransmission of 5-HT and norepinephrine, thus participating in mood and behavior regulation processes (63).

In summary, our current study preliminarily predicted that the mechanism of action of the herb pair of prepared Rebmannia root-Chinese arborvitae kernel in treating anxiety disorders involves multiple ingredients, multiple targets, and multiple pathways. It was found that the herb pair may exert its anxiolytic effect via biological processes such as neuroplasticity, signal transduction, hormone regulation, and inflammatory response through neuroactive ligand-receptor interaction, serotonergic synapse, PI3K/ AKT/mTOR signaling pathway, MAPK signaling pathway, oxytocin signaling pathway, estrogen signaling pathway, and PPAR signaling pathway.

\section{Acknowledgments}

Funding: Grants supported this study from the National Natural Science Foundation of China (No.81774249).

\section{Footnote}

Reporting Checklist: The authors have completed the MDAR checklist. Available at http://dx.doi.org/10.21037/apm-21-531

Conflicts of Interest: All authors have completed the ICMJE uniform disclosure form (available at http://dx.doi. org/10.21037/apm-21-531). The authors have no conflicts of interest to declare.

Ethical Statement: The authors are accountable for all aspects of the work in ensuring that questions related to the accuracy or integrity of any part of the work are appropriately investigated and resolved. The study was conducted in accordance with the Declaration of Helsinki (as revised in 2013).
Open Access Statement: This is an Open Access article distributed in accordance with the Creative Commons Attribution-NonCommercial-NoDerivs 4.0 International License (CC BY-NC-ND 4.0), which permits the noncommercial replication and distribution of the article with the strict proviso that no changes or edits are made and the original work is properly cited (including links to both the formal publication through the relevant DOI and the license). See: https://creativecommons.org/licenses/by-nc-nd/4.0/.

\section{References}

1. Bandelow B, Baldwin D, Abelli M, et al. Biological markers for anxiety disorders, OCD and PTSD: A consensus statement. Part II: Neurochemistry, neurophysiology and neurocognition. World J Biol Psychiatry 2017;18:162-214.

2. Stein DJ, Scott KM, de Jonge P, Kessler RC. Epidemiology of anxiety disorders: from surveys to nosology and back. Dialogues Clin Neurosci 2017;19:127-36.

3. Huang $\mathrm{Y}$, Wang $\mathrm{Y}$, Wang $\mathrm{H}$, et al. Prevalence of mental disorders in China: a cross-sectional epidemiological study. Lancet Psychiatry 2019;6:211-24.

4. Association AP. Diagnostic and statistical manual of mental disorders. BMC Med 2013;17:133-7. https://www. psychiatry.org/psychiatrists/practice/dsm

5. Shi L, Lu ZA, Que JY, et al. Prevalence of and Risk Factors Associated With Mental Health Symptoms Among the General Population in China During the Coronavirus Disease 2019 Pandemic. JAMA Netw Open 2020;3:e2014053.

6. Huang C, Huang L, Wang Y, et al. 6-month consequences of COVID-19 in patients discharged from hospital: a cohort study. Lancet 2021;397:220-32.

7. Liu L, Liu C, Wang Y, et al. Herbal Medicine for Anxiety, Depression and Insomnia. Curr Neuropharmacol 2015;13:481-93.

8. Hou L, Li XY, Huang NN, et al. Internet pharmacology analysis of mechanism of Sini Powder in intervening anxiety disorder. Chinese Traditional and Herbal Drugs 2019;50:5154-61.

9. Shahrajabian $\mathrm{MH}$, Sun $\mathrm{W}$, Soleymani A, et al. Traditional herbal medicines to overcome stress, anxiety and improve mental health in outbreaks of human coronaviruses. Phytother Res 2020. [Epub ahead of print]. doi: 10.1002/ ptr.6888.

10. Yan ZJ. Anxiety Treated through Emotion and Will Differentiation. Shandong Journal of Traditional Chinese 
Medicine 2017;36:443-5.

11. Berger SI, Iyengar R. Network analyses in systems pharmacology. Bioinformatics 2009;25:2466-72.

12. Huang L, Wei XY, Lin X, et al. Study on the therapeutic mechanism of COVID-19(COVID-19) based on network pharmacology and bioinformatics technology. Pharmacology and Clinics of Chinese Materia Medica,2020;36:54-64.

13. Ru J, Li P, Wang J, et al. TCMSP: a database of systems pharmacology for drug discovery from herbal medicines. J Cheminform 2014;6:13.

14. Song ZY, Wang SS, He X, et al. Study the Mechanism of Acorus tatarinowii on Neurodegenerative Diseases by Network Pharmacology. Journal of Hunan University of Chinese Medicine 2017;37:848-55.

15. Kim S, Chen J, Cheng T, et al. PubChem in 2021: new data content and improved web interfaces. Nucleic Acids Res 2021;49:D1388-95.

16. Gfeller D, Grosdidier A, Wirth M, et al. Swiss TargetPrediction: a web server for target prediction of bioactive small molecules. Nucleic Acids Res 2014;42:W32-8.

17. Shannon P, Markiel A, Ozier O, et al. Cytoscape: a software environment for integrated models of biomolecular interaction networks. Genome Res 2003;13:2498-504.

18. Piñero J, Queralt-Rosinach N, Bravo À, et al. DisGeNET: a discovery platform for the dynamical exploration of human diseases and their genes. Database (Oxford) 2015;2015:bav028.

19. Amberger JS, Bocchini CA, Schiettecatte F, et al. OMIM. org: Online Mendelian Inheritance in Man (OMIM®), an online catalog of human genes and genetic disorders. Nucleic Acids Res 2015;43:D789-98.

20. Law V, Knox C, Djoumbou Y, et al. DrugBank 4.0: shedding new light on drug metabolism. Nucleic Acids Res 2014;42:D1091-7.

21. Safran M, Chalifa-Caspi V, Shmueli O, et al. Human Gene-Centric Databases at the Weizmann Institute of Science: GeneCards, UDB, CroW 21 and HORDE. Nucleic Acids Res 2003;31:142-6.

22. Davis AP, Grondin CJ, Johnson RJ, et al. Comparative Toxicogenomics Database (CTD): update 2021. Nucleic Acids Res 2021;49:D1138-43.

23. Szklarczyk D, Gable AL, Lyon D, et al. STRING v11: protein-protein association networks with increased coverage, supporting functional discovery in genome- wide experimental datasets. Nucleic Acids Res 2019;47:D607-13.

24. Sartori SB, Singewald N. Novel pharmacological targets in drug development for the treatment of anxiety and anxietyrelated disorders. Pharmacol Ther 2019;204:107402.

25. Zhou Y, Zhou B, Pache L, et al. Metascape provides a biologist-oriented resource for the analysis of systemslevel datasets. Nat Commun 2019;10:1523.

26. Shimada-Sugimoto M, Otowa T, Hettema JM. Genetics of anxiety disorders: Genetic epidemiological and molecular studies in humans. Psychiatry Clin Neurosci 2015;69:388-401.

27. Lan JQ, Zhao CY, Wu L, et al. Evaluation of non-clinical anxiety disorder models. Acta Pharmaceutica Sinica 2021;01:1-33

28. Cui Y, Feng J, Wang H, et al. Behavioral experiment on anti-anxiety effects of prepared rehmannia root on mice. Chinese Journal of Clinical Rehabilitation 2006;43:61-3.

29. Tallima H, El Ridi R. Arachidonic acid: Physiological roles and potential health benefits - A review. J Adv Res 2017;11:33-41.

30. Haque MN, Hannan MA, Dash R, et al. The potential LXR $\beta$ agonist stigmasterol protects against hypoxia/ reoxygenation injury by modulating mitophagy in primary hippocampal neurons. Phytomedicine 2021;81:153415.

31. Aguirre-Hernández E, Rosas-Acevedo H, Soto-Hernández $\mathrm{M}$, et al. Bioactivity-guided isolation of beta-sitosterol and some fatty acids as active compounds in the anxiolytic and sedative effects of Tilia americana var. mexicana. Planta Med 2007;73:1148-55.

32. López-Rubalcava C, Piña-Medina B, Estrada-Reyes R, et al. Anxiolytic-like actions of the hexane extract from leaves of Annona cherimolia in two anxiety paradigms: possible involvement of the GABA/benzodiazepine receptor complex. Life Sci 2006;78:730-7.

33. Zhao D, Zheng L, Qi L, et al. Structural Features and Potent Antidepressant Effects of Total Sterols and $\beta$-sitosterol Extracted from Sargassum horneri. Mar Drugs 2016;14:123.

34. Galbusera C, Facheris M, Magni F, et al. Increased susceptibility to plasma lipid peroxidation in Alzheimer disease patients. Curr Alzheimer Res 2004;1:103-9.

35. Ntais C, Polycarpou A. Association of the endothelial nitric oxide synthase (NOS3) Glu298Asp gene polymorphism with the risk of Alzheimer's disease-- a meta-analysis. J Neurol 2005;252:1276-8.

36. Guo LJ, He ZC, Li YJ, et al. The relationship between 
$\alpha$-synuclein,A $\mathrm{A} 1-42$,EGF and the degree of illness and cognitive dysfunction in patients with severe parkinson's disease. Journal of Tropical Medicine 2020;20:1573$1576+1584$.

37. Yang H, Wang H, Shu Y, et al. miR-103 Promotes Neurite Outgrowth and Suppresses Cells Apoptosis by Targeting Prostaglandin-Endoperoxide Synthase 2 in Cellular Models of Alzheimer's Disease. Front Cell Neurosci 2018;12:91.

38. Zhuang J, Chen Z, Cai P, et al. Targeting MicroRNA125b Promotes Neurite Outgrowth but Represses Cell Apoptosis and Inflammation via Blocking PTGS2 and CDK5 in a FOXQ1-Dependent Way in Alzheimer Disease. Front Cell Neurosci 2020;14:587747.

39. Frémin C, Saba-El-Leil MK, Lévesque K, et al. Functional Redundancy of ERK1 and ERK2 MAP Kinases during Development. Cell Rep 2015;12:913-21.

40. Antypa N, Souery D, Tomasini M, et al. Clinical and genetic factors associated with suicide in mood disorder patients. Eur Arch Psychiatry Clin Neurosci 2016;266:181-93.

41. Rudko OI, Tretiakov AV, Naumova EA, et al. Role of PPARs in Progression of Anxiety: Literature Analysis and Signaling Pathways Reconstruction. PPAR Res 2020;2020:8859017.

42. Ryan J, Scali J, Carrière I, et al. Estrogen receptor gene variants are associated with anxiety disorders in older women. Psychoneuroendocrinology 2011;36:1582-6.

43. Sundermann EE, Maki PM, Bishop JR. A review of estrogen receptor alpha gene (ESR1) polymorphisms, mood, and cognition. Menopause 2010;17:874-86.

44. Marcinkiewcz CA, Mazzone CM, D'Agostino G, et al. Serotonin engages an anxiety and fear-promoting circuit in the extended amygdala. Nature 2016;537:97-101.

45. Iurescia S, Seripa D, Rinaldi M. Looking Beyond the 5-HTTLPR Polymorphism: Genetic and Epigenetic Layers of Regulation Affecting the Serotonin Transporter Gene Expression. Mol Neurobiol 2017;54:8386-403.

46. Pulido R. PTEN Inhibition in Human Disease Therapy. Molecules 2018;23:285.

47. Sánchez-Puelles C, Calleja-Felipe M, Ouro A, et al. PTEN Activity Defines an Axis for Plasticity at CorticoAmygdala Synapses and Influences Social Behavior. Cereb Cortex 2020;30:505-24.

48. Papassotiropoulos A, Gerhards C, Heck A, et al. Human genome-guided identification of memory-modulating drugs. Proc Natl Acad Sci U S A 2013;110:E4369-74.
49. Lu Y, Yang J, Sun J, et al. mRNA and miRNA profiles in the nucleus accumbens are associated with psychological stress-induced susceptible and resilient mice. Pharmacol Biochem Behav 2020;199:173062.

50. Franke TF. PI3K/Akt: getting it right matters. Oncogene 2008;27:6473-88.

51. Duman RS, Voleti B. Signaling pathways underlying the pathophysiology and treatment of depression: novel mechanisms for rapid-acting agents. Trends Neurosci 2012;35:47-56.

52. Zhang ZN, Liang LY, Lian JH, et al. PI3K/AKT/m TOR Signaling Pathway in Central Nervous System. The Journal of Practical Medicine 2020,36:689-94.

53. Lipton JO, Sahin M. The neurology of mTOR. Neuron 2014;84:275-91.

54. Li N, Lee B, Liu RJ, et al. mTOR-dependent synapse formation underlies the rapid antidepressant effects of NMDA antagonists. Science 2010;329:959-64.

55. Wang A, Zou X, Wu J, et al. Early-Life Stress Alters Synaptic Plasticity and mTOR Signaling: Correlation With Anxiety-Like and Cognition-Related Behavior. Front Genet 2020;11:590068.

56. Won, E.; Kim, Y. K. Neuroinflammation-Associated Alterations of the Brain as Potential Neural Biomarkers in Anxiety Disorders. Int. J. Mol. Sci. 2020;21:6546.

57. Albert-Gascó H, Ros-Bernal F, Castillo-Gómez E, et al. MAP/ERK Signaling in Developing Cognitive and Emotional Function and Its Effect on Pathological and Neurodegenerative Processes. Int J Mol Sci 2020;21:4471.

58. Jurek B, Slattery DA, Maloumby R, et al. Differential contribution of hypothalamic MAPK activity to anxietylike behaviour in virgin and lactating rats. PLoS One 2012;7:e37060.

59. Ding L, Zhang C, Masood A, et al. Protective effects of phosphodiesterase 2 inhibitor on depression- and anxietylike behaviors: involvement of antioxidant and antiapoptotic mechanisms. Behav Brain Res 2014;268:150-8.

60. Delhaye S, Bardoni B. Role of phosphodiesterases in the pathophysiology of neurodevelopmental disorders. Mol Psychiatry 2021. [Epub ahead of print]. doi: 10.1038/ s41380-020-00997-9.

61. Chen L, Zhang X, Hu C, et al. Regulation of GABAA and 5-HT Receptors Involved in Anxiolytic Mechanisms of Jujube Seed: A System Biology Study Assisted by UPLCQ-TOF/MS and RT-qPCR Method. Front Pharmacol 2020;11:01320.

62. Xin F, Zhou X, Dong D, et al. Oxytocin Differentially 
Modulates Amygdala Responses during Top-Down and Bottom-Up Aversive Anticipation. Adv Sci (Weinh) 2020;7:2001077.

63. Bauer M, London ED, Silverman DH, et al. Thyroid, brain and mood modulation in affective disorder: insights

Cite this article as: Liu PL, Song AR, Dong CD, Chu Q, Xu BL, Liu JM, Yan ZJ. Network pharmacology study on the mechanism of the herb pair of prepared Rebmannia rootChinese arborvitae kernel for anxiety disorders. Ann Palliat Med 2021;10(3):3313-3327. doi: 10.21037/apm-21-531 from molecular research and functional brain imaging. Pharmacopsychiatry 2003;36 Suppl 3:S215-21.

(English Language Editor: J. Gray) 